Technical notes

\title{
Study of dental prostheses influence in radiation therapy
}

\author{
C. De Conto ${ }^{\mathrm{a}, \mathrm{b}, *}$, R. Gschwind ${ }^{\mathrm{a}}$, E. Martin ${ }^{\mathrm{b}}$, L. Makovicka ${ }^{\mathrm{a}}$ \\ a IRMA/LCPR-AC/CE, UMR 6249 CNRS, Université de Franche-Comté, BP 71427, 25211 Montbéliard, France \\ b Centre Hospitalier Belfort Montbéliard, Service d'Oncologie et de Radiothérapie, site du Mittan, 56 boulevard du Maréchal Juin, 25209 Montbéliard, France
}

\section{A R T I C L E I N F O}

\section{Article history:}

Received 7 May 2012

Received in revised form

12 December 2012

Accepted 4 March 2013

Available online 11 April 2013

\section{Keywords:}

Dental prostheses

Radiotherapy

Dose distribution

BEAMnrc/EGSnrc codes

\begin{abstract}
A B S T R A C T
Dental prostheses made of high density material contribute to modify dose distribution in head and neck cancer treatment. Our objective is to quantify dose perturbation due to high density inhomogeneity with experimental measurements and Monte Carlo simulations.

Firstly, measurements were carried in a phantom representing a human jaw with thermoluminescent detectors (GR200A) and EBT2 Gafchromic films in the vicinity of three samples: a healthy tooth, a tooth with amalgam and a $\mathrm{Ni}-\mathrm{Cr}$ crown, irradiated in clinical configuration. Secondly, Monte Carlo simulations (BEAMnrc code) were assessed in an identical configuration.

Experimental measurements and simulation results confirm the two well-known phenomena: firstly the passage from a low density medium to a high density medium induces backscattered electrons causing a dose increase at the interface, and secondly, the passage from a high density medium to a low density medium creates a dose decrease near the interface. So, the results show a $1.4 \%$ and $23.8 \%$ backscatter dose rise and attenuation after sample of $26.7 \%$ and $10.9 \%$ respectively for tooth with amalgam and crown compared to the healthy tooth.

Although a tooth with amalgam has a density of about $12-13$, the changes generated are not significant. However, the results for crown (density of 8 ) are very significant and the discordance observed may be due to calculation point size difference $0.8 \mathrm{~mm}$ and $0.25 \mathrm{~mm}$ respectively for TLD and Monte Carlo. The use of Monte Carlo simulations and experimental measurements provides objective evidence to evaluate treatment planning system results with metal dental prostheses.
\end{abstract}

(c) 2013 Associazione Italiana di Fisica Medica. Published by Elsevier Ltd. All rights reserved.

\section{Introduction}

Head and neck cancers represent about $20 \%$ of the cancers treated by radiation therapy in our institution. Amongst these patients, most of them have non-removable dental prostheses. Two types are mainly represented: an amalgam that is a material allowing to replace a hole left by caries and a crown that repairs a deteriorated tooth or covers an implant when a tooth is missing. All materials with a density higher than the human body density (like metal) are considered as high density, contrary to air which is a low density media.

The effect of low density is widely studied especially in AAPM report no 85 [1], so air is generally correctly taken into account. For high density implants, several papers about hip prostheses exist, mainly AAPM report no 81 [2] and work from our laboratory [3,4],

\footnotetext{
* Corresponding author. IRMA/LCPR-AC/CE, UMR 6249 CNRS, Université de Franche-Comté, BP 71427, 25211 Montbéliard, France. Tel.: +33 3819946 75; fax: +33 381994673 .

E-mail addresses: cdeconto@univ-fcomte.fr (C. De Conto), regine.gschwind@ univ-fcomte.fr (R. Gschwind).
}

which propose several recommendations. For a pelvic treatment with hip prostheses despite artifacts and attenuation related to metal, beam setup can be adjusted to avoid passing through the prosthesis in order to deliver the correct dose to the target.

However, these recommendations are difficult to apply for a head and neck cancer treatment because (i) there are usually more than one or two prostheses, (ii) the resulting artifacts cover entire CT image, (iii) the target is often very close to prostheses, inside artifacts and (iv) in this small area, it is difficult to avoid prostheses (Fig. 1).

Several aspects of dental prostheses were previously investigated. Farahani et al. and Russell et al. studied doses at interfaces [5,6], teams of Nadrowitz, Beyzadeaglu and Thilmann evaluated the beam angle influence [7-9]. Thilmann et al. achieve in vivo measurements with ${ }^{60} \mathrm{Co}$ [9]. These works were done in simple conditions using a single sample by experimental measurements. A study suggests the use of cotton or water equivalent protection around teeth, like dental protection for sportsmen $[10,11]$ but it is not easy and comfortable for patients so it is never setup in clinical.

The most recent publications use Monte Carlo methods where simulations are compared with experimental measurements 


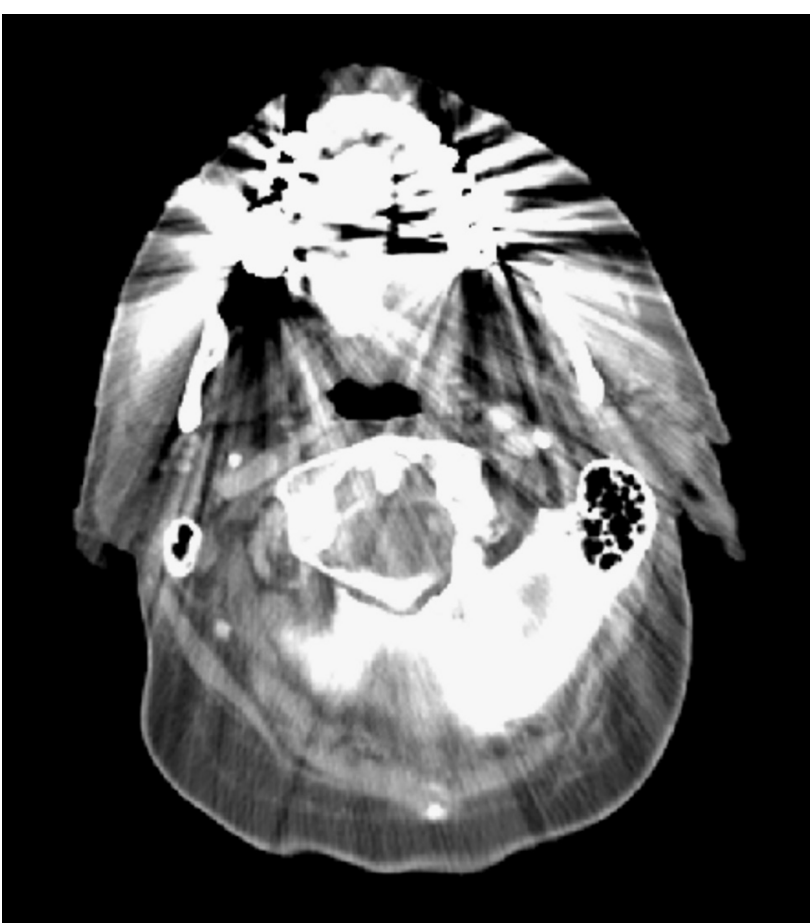

Figure 1. A CT-slice with artifacts due to dental prostheses.

[12-15]. Palleri et al. and Spiridovich et al. [14,16] also compared Monte Carlo with commercial Pinnacle Treatment Planning System (TPS), Philips, and superposition algorithm, both showing weaknesses of these systems. On their side, Webster et al. [17] studied the efficiency of different artifact corrections on CT images on 15 patients.

In this study, we focus on the evaluation of one sample influence in a $6 \mathrm{MV}$ photon beam (i) by experimental measurements using thermoluminescent detector (TLD) in homemade phantom, (ii) by Monte Carlo simulations using BEAMnrc code in which the phantom was modeled. Our goal is to compare with clinical treatment planning system (TPS) calculations performed on CT images without artifacts correction.

\section{Materials and methods}

In order to estimate the dose at interfaces, three systems are compared: experimental measurements, simulations by Monte Carlo method and treatment planning system used in clinical with a homemade phantom.

\section{Phantom}

A phantom was specially built and composed of five $12 \mathrm{~cm} \times 24$ $\mathrm{cm} \times 1 \mathrm{~cm}$-thick slabs of PMMA (Polymethyl Methacrylate), as well
Table 1

Composition, density and effective atomic number of different materials.

\begin{tabular}{lllc}
\hline Material & Composition & Density & $Z_{\text {eff }}[18]$ \\
\hline Water/tissue & $\mathrm{H}_{2} \mathrm{O} / \mathrm{H}, \mathrm{C}, \mathrm{N}, \mathrm{O}$ & 1 & 7.5 \\
Bone & $\mathrm{H}, \mathrm{C}, \mathrm{N}, \mathrm{O}, \mathrm{Mg}, \mathrm{P}, \mathrm{S}, \mathrm{Ca}, \mathrm{Zn}$ & 1.85 & 11.8 \\
Tooth & $\mathrm{H}, \mathrm{Na}, \mathrm{Mg}, \mathrm{C}, \mathrm{P}, \mathrm{O}, \mathrm{Ca}$ & 2.2 & 8.5 \\
PMMA & Polymer & 1.19 & 6.5 \\
PVDF & Polymer & 1.78 & 9 \\
Bolus & Silicon & 1.05 & 7.3 \\
Amalgam & $50 \% \mathrm{Hg}, 30 \% \mathrm{Ag}, \mathrm{Cu}, \mathrm{Sn}, \mathrm{Zn}$ & $12-13$ & 67.4 \\
Crown & $59.5 \% \mathrm{Co}, 31.5 \% \mathrm{Cr}, 5 \% \mathrm{Mo}, 2 \% \mathrm{Si}, 1 \% \mathrm{Mn}$ & 8.8 & 27.1 \\
\hline
\end{tabular}

as three central slabs perforated in a U-shaped jaw and dental arch (see Fig. 2). Two slabs are replaced by PVDF (PolyVinyliDene Fluoride) and the last slab allows putting a sample and detectors in bolus material.

PMMA, PVDF and bolus are, respectively, water, bone and water equivalent materials, which have a close physical density and a close effective atomic number. A medium is equivalent to another if it can reproduce a cross section and if their collision stopping powers are similar. The last column in Table 1 gives the effective atomic number of materials.

\section{Samples}

Three real tooth samples were used independently: a healthy tooth, a tooth with amalgam (the quantity of amalgam is represented in hatched area in Fig. 2) and a crown. They represent the two most frequent metallic materials.

Table 1 gives the composition, the density and the effective atomic number $\left(Z_{\mathrm{eff}}\right)$ of various materials used in this study. $Z_{\mathrm{eff}}$ was determined according to Mayneord formula [18].

One can notice from the density data, that the mean density value for a tooth is slightly greater than bone but relatively close. Therefore it is taken into account in Hounsfield unit and so in TPS. The choice is done to use the tooth as a reference.

\section{Experimental measurements}

Experimental measurements are performed using thermoluminescent detectors (TLD), GR200A type $(4.5 \mathrm{~mm}$ in diameter and $0.8 \mathrm{~mm}$ thick) calibrated. The phantom permits the positioning of four TLD perpendiculars to the beam direction: one at each interface, one at $5 \mathrm{~mm}$ before the sample and one at $5 \mathrm{~mm}$ after the sample (see Fig. 3).

\section{Simulations}

The Monte Carlo simulations are achieved using the OMEGA/ BEAMnrc code V4 (2009) [19,20] specific to radiotherapy. 6 MV photon beam of Clinac 2100C, VARIAN linear accelerator was modeled in BEAMnrc module to create a phase space at $90 \mathrm{~cm}$ of the source. The phantom described in Section 2.1 was modeled voxel by
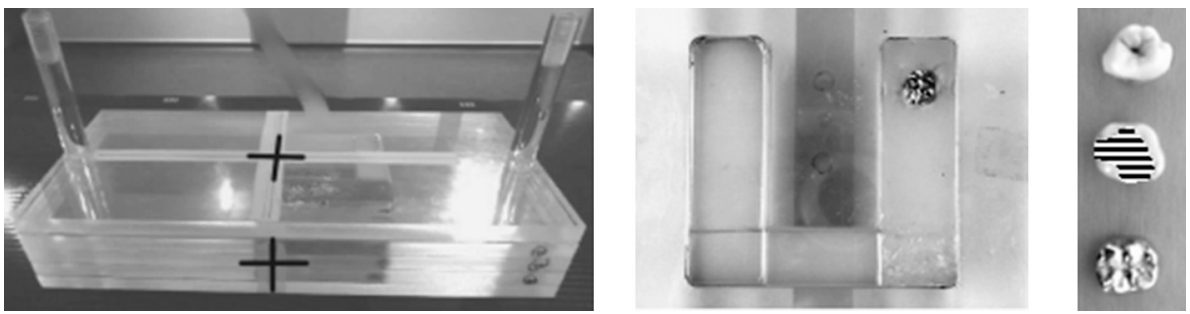

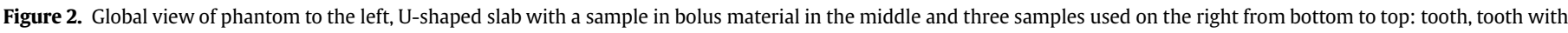
amalgam (quantity of amalgam in the tooth is represented approximately in hatched area) and crown. 


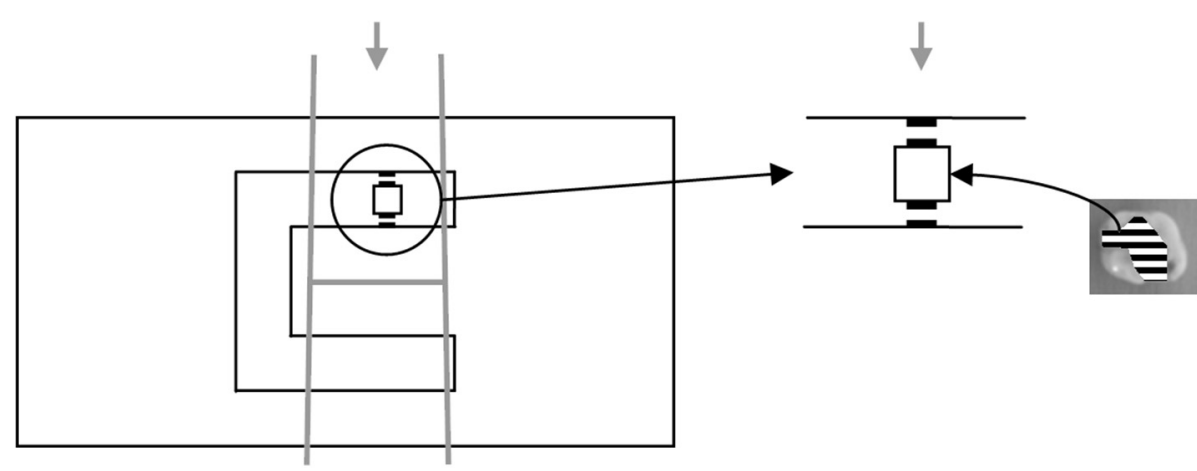

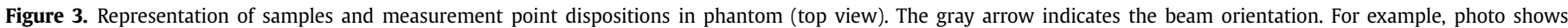
amalgam orientation in the beam.

voxel in Cartesian geometry. A material is assigned to every voxel. The peripheral voxels are $5 \mathrm{~mm} \times 5 \mathrm{~mm} \times 5 \mathrm{~mm}$ in order to obtain a gain for computation time. The voxels in the region of interest are smaller $(0.25 \mathrm{~mm} \times 0.25 \mathrm{~mm} \times 0.25 \mathrm{~mm})$ to improve the result accuracy. The cross section of amalgam and crown was created in PEGS4 module while tooth was approximated with pyrex.

The dose distribution was determined by DOSXYZnrc module [21] using phase space, cross sections and modeled phantom. One billion of particles were simulated up to cut off energies of $521 \mathrm{keV}$ and $10 \mathrm{keV}$, for electrons and photons, respectively. The uncertainty is below $3 \%$ in the worst case (i.e. tooth with amalgam and in the smallest voxels).

\section{Treatment planning system (TPS)}

Oncentra Master Plan (OMP) from Nucletron v3.1 sp3 [22], is the Treatment Planning System (TPS) used to plan treatments. Two algorithms were implemented: Pencil Beam [23] and Collapsed Cone [24]. Each algorithm uses a specific inhomogeneity correction method, presented below. These corrections can be selected or not by the user. If not, the medium in which calculation were achieved is water.

The method of inhomogeneity correction used in Pencil Beam algorithm uses the equivalent path length method for primary dose and $1 \mathrm{D}$ convolution correction for scattered dose respectively given by Equations (1) and (2).

$z_{\text {eq }}=z_{\text {geom }} \cdot \mu_{\text {medium }} / \mu_{\text {water }}$

where $z_{\text {geom }}$ is thickness of the crossed media in $\mathrm{cm}, \mu_{\text {medium }}$ is the attenuation coefficient of the media in $\mathrm{cm}^{-1}$ and $\mu_{\text {water }}$ is the attenuation coefficient of water in $\mathrm{cm}^{-1}$.

$\mathrm{CF}=z_{\text {eq }} / z_{\text {geom }} \cdot \exp \left(\mu_{80 \%}\left(z_{\text {eq }}-z_{\text {geom }}\right)\right)$

where $z_{\mathrm{eq}}$ is the equivalent path length in $\mathrm{cm}, z_{\text {geom }}$ is the thickness of the crossed media in $\mathrm{cm}$ and $\mu_{80 \%}$ is $80 \%$ of the attenuation coefficient of the primary beam in $\mathrm{cm}^{-1}$.

For Collapsed Cone algorithm, inhomogeneity correction is performed using kernel rescaled which provide better accuracy [24].

The phantom was scanned like a patient, and the same beam configuration was planned. The calculation matrix is $0.5 \mathrm{~cm} \times$ $0.25 \mathrm{~cm} \times 0.5 \mathrm{~cm}$ (where $0.25 \mathrm{~cm}$ is the slice thickness). The use of an extensive scale is not possible but the maximum value of Hounsfield unit is considered (like iron) with a density equals to 7.9. However, it is possible to assign a density value to a volume (beforehand delineated), the maximum possible value is 2.8 (given by the crown). In order to overcome the artifacts, a density value of 1 must be assigned to the rest of the volume.

\section{Irradiation conditions}

The dose is delivered at the isocentre at a $6 \mathrm{~cm}$ depth of a phantom with a $6 \mathrm{MV}$ X-ray lateral beam, perpendicular to the sample (see Fig. 3) similarly to beams used for a head and neck cancer treatment. The field has a $5 \mathrm{~cm} \times 5 \mathrm{~cm}$ dimension in SID technique (Source Isocenter Distance of $100 \mathrm{~cm}$ ).

The purpose is to deliver $2 \mathrm{~Gy}$ at the isocenter. For a homogenous water phantom configuration, this dose corresponds to 191 monitor units (MU) for this treatment device. Therefore, this value was used in all sample configurations for experimental measurements and TPS calculations.

\section{Results and discussion}

The influence of high density prostheses compared to healthy teeth is determined with three different methods: experimental measurements with TLD, Monte Carlo simulation and Pencil Beam algorithm with inhomogeneity correction are given in Table 2 for a $6 \mathrm{MV}$ photon beam. The uncertainties of these methods are below $2 \%, 3 \%(2 \%)$ and $4 \%$, respectively, for TLD, BEAMnrc with amalgam (BEAMnrc for others samples) and OMP TPS.

Figure 4 presents the results of clinical algorithms compared to TLD for the crown. Figure 5 shows the Monte Carlo simulations results for the three samples. Figure 6 presents the dose of the three samples using algorithm Pencil Beam clinical with inhomogeneity correction. The results are discussed according two area of interest (i) the first area is before the sample where we search to observe the backscatter phenomenon and (ii) the second area is after the sample where the dose is attenuated.

\section{First interface: backscatter evaluation}

For the crown, only Monte Carlo simulations highlight backscatter of electrons with a dose increase of $+23.7 \%$ compared with

\section{Table 2}

Results with TLD (2\%), Monte Carlo simulation (3\%) and clinical system (4\%) for tooth with amalgam and crown compared with healthy tooth (uncertainty in brackets).

\begin{tabular}{|c|c|c|c|c|c|c|}
\hline \multirow{2}{*}{$\begin{array}{l}\text { Dose difference } \\
\text { at the interface } \\
\text { compared with } \\
\text { healthy tooth } \\
\text { (in \%) }\end{array}$} & \multicolumn{3}{|c|}{ Tissue/sample interface } & \multicolumn{3}{|c|}{ Sample/tissue interface } \\
\hline & TLD & Monte Carlo & TPS $^{\mathrm{a}}$ & TLD & Monte Carlo & TPS $^{\mathrm{a}}$ \\
\hline $\begin{array}{l}\text { Tooth with } \\
\text { amalgam }\end{array}$ & $-4.26 \%$ & $+1.43 \%$ & $+0.22 \%$ & $-0.37 \%$ & $-26.72 \%$ & $-1.81 \%$ \\
\hline Crown & $+2.13 \%$ & $+23.82 \%$ & $+0.28 \%$ & $-13.6 \%$ & $-10.95 \%$ & $-1.70 \%$ \\
\hline
\end{tabular}

a Pencil Beam algorithm with inhomogeneity correction. 


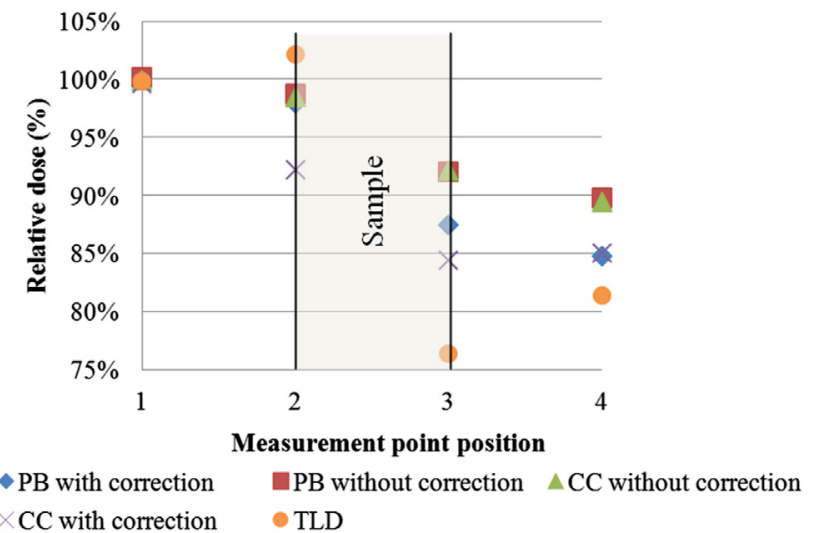

Figure 4. Comparison between Pencil Beam and collapsed cone with and without inhomogeneity correction for crown where position 1 is $5 \mathrm{~mm}$ before sample, position 2 is at interface tissue/sample, position 3 is at interface sample/tissue and position 4 is $5 \mathrm{~mm}$ after the sample (uncertainty of $4 \%$ ).

tooth. The $0.25 \mathrm{~mm}$ dimension grid can explain this results that is not observed with TLD $(0.8 \mathrm{~mm})$ and TPS $(5 \mathrm{~mm})$. The backscatter phenomenon happens only along few micrometers and, more importantly, with high density and high photon energy [1].

For the tooth with amalgam, there are no differences with the tooth using the three methods. These results are the consequences of the sample configuration: the amalgam is surrounded by tooth so the interface with amalgam is inside the tooth and not accessible for measurement. However, the depth dose profile obtained with Monte Carlo simulations shows the backscatter inside the tooth.

These results are consistent with the works leads by Farahani, Russell and Ravikumar teams [5,6,25] who concentrated their researches on backscattered experimental measurements. They showed a dose increase up to $25 \%, 30 \%, 60 \%$ and $70 \%$, respectively, for tooth, $\mathrm{NiCr}$ alloy, gold alloy and $\mathrm{AgHg}$ amalgam compared to polystyrene.

\section{Second interface and beyond: attenuation evaluation}

For tooth with amalgam, only Monte Carlo simulations show a sharp attenuation, this is not confirmed by other methods. This result is probably caused by a difference in the composition between the sample and the material defined in the simulation. These results must be confirmed in future study by specifying composition.

For crown, the attenuation was significant, the evaluated underdosages were $10.6 \%, 17.1 \%, 1.7 \%$ and $3.7 \%$, respectively, for

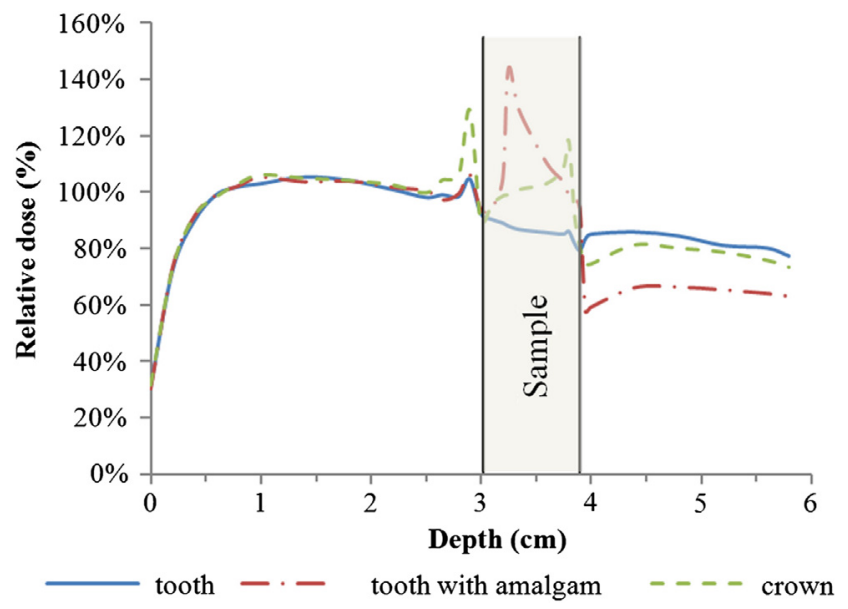

Figure 5. Results obtained by Monte Carlo simulation for tooth, tooth with amalgam and crown (uncertainty below $3 \%$ ).

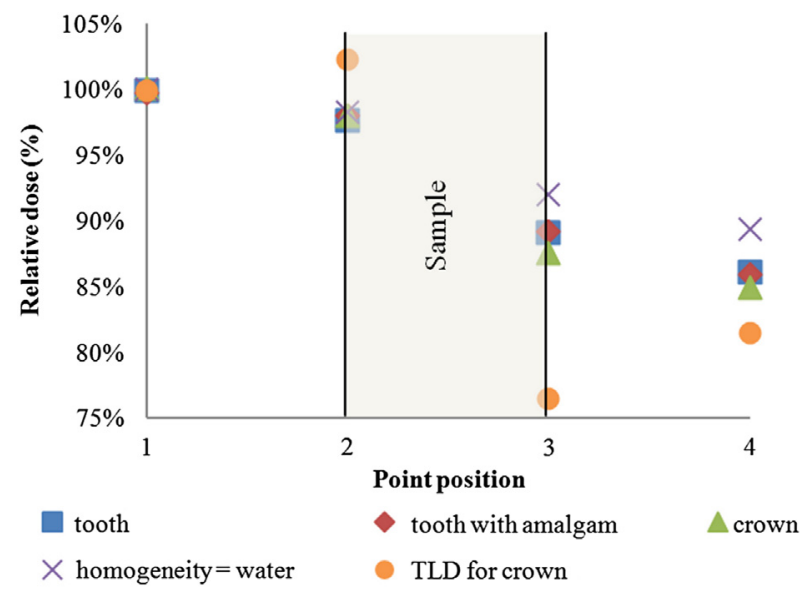

Figure 6. Results obtained for tooth, tooth with amalgam and crown for Pencil Beam algorithm with inhomogeneity correction compared with Pencil Beam algorithm without inhomogeneity correction (i.e. homogeneity water medium) and compared with TLD measurement for the crown where position 1 is $5 \mathrm{~mm}$ before sample, position 2 is at interface tissue/sample, position 3 is at interface sample/tissue and position 4 is $5 \mathrm{~mm}$ after sample (uncertainty of $4 \%$ ).

Monte Carlo, TLD, Pencil Beam with inhomogeneity correction and Collapsed Cone with inhomogeneity correction.

We can notice on Fig. 4 that PB and CC algorithms, without inhomogeneity correction, give the same results since both computations are performed for water homogenous medium. When inhomogeneity correction was selected, CC is closer to experimental measurements (TLD) than PB. However the attenuation is underestimated by around $10.5 \%$ and $14.6 \%$ respectively for CC and $\mathrm{PB}$.

Moreover, for crown and amalgam ( $\mathrm{Hg}-\mathrm{Ag}$ alloy), we can observe with MC method a non-negligible attenuation persistent at $2 \mathrm{~cm}$ after sample respectively of $4.9 \%$ and $18.1 \%$, exactly where the target volume is.

For further studies, Monte Carlo simulation will be taken as a reference in a first approach, instead of initiating experimental measurements, which have complex setup and are time consuming. Many studies $[12,16]$ validated BEAMnrc as a reference for radiotherapy up to iron material $(d=7.9)$.

For CT images, Fig. 7 shows the results of two delineated methods: first, the crown is delineated and a 2.4 density was attributed (blue triangle). In the second case, the crown is also delineated keeping the

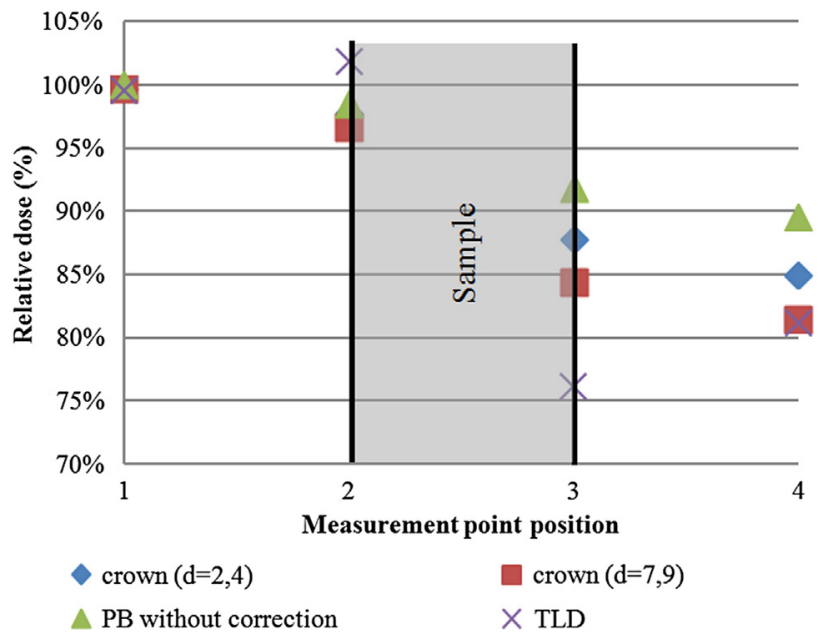

Figure 7. Results obtained for crown with maximal manual correction of density $(d=2.4)$, crown with density corresponding to Hounsfield units compared with water (i.e. the same images calculate with Pencil Beam algorithm without inhomogeneity correction) and compared with TLD measurements. 
HU value ( $3071 \mathrm{HU}$ corresponding to 7.9 density) and a density of 1 is attributed to the rest of the volume. The TLD measurements are also reported so we can observe that the second delineated, solution is closest, but the attenuation is underestimated of $9.7 \%$. In this case, the delineated time consumption is not evaluated. Since the conditions are simple, these observations provide a first approach on density correction solutions.

\section{Conclusion}

The influence of dental prostheses was investigated by comparing the results of experimental measurements, Monte Carlo simulations and TPS calculations. The three tested real samples are representative of what is encountered: healthy tooth, tooth with amalgam and crown. The irradiation configuration is a simplification of a head and neck cancer treatment, i.e. a lateral 6 MV photon beam.

The backscatter with high density material is observed and supported by many studies. A proposed solution is to protect using water equivalent material around teeth but it is difficult to apply in practical. Whereas the attenuation is demonstrated, the consequences on the target volume are undeveloped, and especially the artifacts on CT images used for dose planning in order to treat a cancerous tumor.

In addition to focusing on attenuation phenomenon, the goal of further study will be to compare Monte Carlo simulations and clinical planning system by reproducing head and neck cancer treatment on CT patient images with or without artifacts. This comparison will evaluate, in practical, the dose distribution modification and if the differences on treatment are significant, while contributing to an evaluation of available methods for density reattributions.

\section{Acknowledgments}

We would like to thank La ligue contre le cancer, Pays de Montbéliard Agglomération, Cancéropôle Grand Est for their financial support and Mesocentre de l'Université de Franche-Comté for the availability of their technical support for calculations.

\section{References}

[1] Papanikolaou N, Battista JJ, Boyer AL, Kappas C, Klein E, Rock Mackie T, et al Tissue inhomogeneity corrections for megavoltage photon beams, vol. 65 Medical Physics Publishing; August 2004. Report no 85 of AAPM Radiation Therapy Committee Task Group.

[2] Reft C, Alecu R, Das IJ, Gerbi BJ, Keall P, Lief E, et al. Dosimetric considerations for patients with hip prostheses undergoing pelvic irradiation. Report of the AAPM Radiation Therapy Committee Task Group 63. Med Phys 2003;30(6): $1162-82$
[3] Buffard E, Gschwind R, Makovicka L, Martin E, Meunier C, David C. Study of the impact of artificial articulations on the dose distribution under medical irradiation. NIM B229 2005:78-84.

[4] Buffard E, Gschwind R, Makovicka L, David C. Monte Carlo calculations of the impact of hip prosthesis on the dose distribution. NIM B251 2006:9-18.

[5] Farahani M, Eichmiller FC. Metal polysiloxane shields for radiation therapy of maxilla-facial tumors. Med Phys 1991;18(2):273-6.

[6] Russell R, Pillai K, Jones PK. In vitro backscattering from implant materials during radiotherapy. J Prosthetic Dentistry 1996;75(6):626-32.

[7] Nadrowitz R, Feyerabend T. Backscatter dose from metallic materials due to obliquely incident high-energy photon beams. Med Phys 2001;28(6): 959-65.

[8] Beyzadeaglu M, Dirican B, Oysul K, Ozen J, Ucok O. Evaluation of scatter dose of dental titanium implants exposed to photon beams of different energies and irradiation angles in head and neck radiotherapy. Dentomaxillofacial Radiol 2006;35:14-7.

[9] Thilmann C, Adamietz IA, Ramm U, Rahn R, Mose S, Saran F, et al. In vivo dose increase in the presence of dental alloys during ${ }^{60}$ Co-gamma-ray therapy of the oral cavity. Med Dos 1996;21(3):149-54.

[10] Farahani M, Eichmiller FC, McLaughlin WL. Metal polysiloxane shields for radiation therapy of maxilla-facial tumors. Med Phys 1991;18(2):273-8.

[11] Reitemeier B, Reitemeier G, Schmidt A, Schaal W, Blochberger P, Lehmann D, et al. Evaluation of a device for attenuation of electron release from dental restorations in a therapeutic radiation field. J Prosthet Dent 2002;87:323-7.

[12] Sauer OA. Calculation of dose distributions in the vicinity of high-Z interfaces for photon beams. Med Phys 1995;22(10):1685-90.

[13] Krieger T, Sauer OA. Monte Carlo versus pencil beam/collapsed cone dose calculation in a heterogeneous multi-layer phantom. Phys Med Biol 2005;50: 859-68.

[14] Spirydovich S, Papiez L, Langer M, Sandison G, Thai V. High density dental materials and RT planning: comparison of the dose predictions using superposition algorithm and fluence map Monte Carlo method with radiochromic film measurements. Radiother Oncol 2006;81:309-14.

[15] Chin DWH, Treister N, Friedland B, Cormack RA, Tishler RB, Makrigiorgos GM, et al. Effect of dental restorations and prostheses on radiotherapy dose distribution: a Monte Carlo study. J Appl Clin Med Phys 2009;10(1):80-9.

[16] Palleri F, Baruffaldi F, Angelini AL, Ferri A, Spezi E. Monte Carlo characterization of materials for prosthetic implants and dosimetric validation of Pinnacle TPS. NIM B266 2008:5001-6.

[17] Webster GJ, Rowbottom CG, Mackay RI. Evaluation of the impact of dental artefacts on intensity-modulated radiotherapy planning for the head and neck. Radiother Oncol 2009;93:553-8.

[18] Mayneord WV. The significance of the roentgen. Acta Union Int Contre Cancer 1937;2:271-82.

[19] Rogers DWO, Walters B, Kawrakow I. BEAMnrc users manual. NRCC Report PIRS-0509(A)revI 2011.

[20] Kawrakow I, Rogers DWO. The EGSnrc code system: MC simulation of electron and photon transport. Technical Report PIRS-701, NRCC, Ottawa, Canada; 2011.

[21] Walters B, Rogers DWO. DOSXYZnrc users manual. NRCC Report PIRS-794revB 2011.

[22] Oncentra Master Plan v3.1 SP3, Physics and algorithms manual 192.739ENG01 , Nucletron.

[23] Ahnesjö A. Collapsed cone convolution of radiant energy for photon dose calculation in heterogeneous media. Med Phys 1989;16(4):577-92.

[24] Ahnesjö A, Saxner M, Trepp A. A pencil beam model for photon dose calculation. Med Phys 1992;19(2):263-73.

[25] Ravikumar M, Ravichandran R, Sathiyan S, Supe SS. Backscattered dose perturbation effects at metallic interfaces irradiated by high-energy $\mathrm{X}$ and gamma ray therapeutic beams. Strahlenther Onkol 2004;3:173-8. 